ARTIGO ORIGINAL

\title{
Estoque de energia da madeira em áreas sob manejo florestal no Rio Grande do Norte
}

\author{
Energy stock in areas under forest management in the state of Rio Grande do \\ Norte
}

Cynthia Patricia de Sousa Santos ${ }^{1}$ (D), Rosimeire Cavalcante dos Santos ${ }^{1}$ (D), Adailton José Epaminondas de Carvalho ${ }^{2}$ (1), Renato Vinícius Oliveira Castro ${ }^{3}$ (i), Sarah Esther de Lima Costa ${ }^{1}$ (D) Luzia Inês Lopes $^{1}$ (D), Frans Germain Corneel Pareyn ${ }^{4}$ (D), Ananias Francisco Dias Júnior ${ }^{5}$ (1) , Paulo Fernando Trugilho ${ }^{6}$ (D), Nickson Fernandes de Oliveira Carvalho ${ }^{1}$ (D), Mateus Alves de Magalhães ${ }^{7}$ [D

${ }^{1}$ Universidade Federal do Rio Grande do Norte - UFRN, Macaíba, RN, Brasil

${ }^{2}$ Florescer Projetos e Consultoria - FPC, Natal, RN, Brasil

${ }^{3}$ Universidade Federal de São João del-Rei - UFSJ, São João del-Rei, MG, Brasil

${ }^{4}$ Associação Plantas do Nordeste - APNE, Recife, PE, Brasil

${ }^{5}$ Universidade Federal do Espírito Santo - UFES, Jerônimo Monteiro, ES, Brasil

${ }^{6}$ Universidade Federal de Lavras - UFLA, Lavras, MG, Brasil

${ }^{7}$ Universidade Federal de Viçosa - UFV, Viçosa, MG, Brasil

Como citar: Santos, C. P. S., Santos, R. C., Carvalho, A. J. E., Castro, R. V. O., Costa, S. E. L., Lopes, L. I., Pareyn, F. G. C., Dias Júnior, A. F., Trugilho, P. F., Carvalho, N. F. O., \& Magalhães, M. A. (2020). Estoque de energia da madeira em áreas sob manejo florestal no Rio Grande do Norte. Scientia Forestalis, 48(126), e3080. https://doi.org/10.18671/scifor.v48n126.06

\section{Resumo}

O objetivo deste trabalho foi determinar a densidade energética em três diferentes áreas sob manejo florestal sustentável do Rio Grande do Norte (RN). A partir do acesso ao inventário florestal de cada área foi realizada a seleção das espécies com $70 \%$ de representatividade baseada no índice de valor de importância (IVI), assim como o conhecimento sobre o volume real de madeira. De cada espécie, foram abatidas três árvores com características que representavam cada povoamento, e realizadas amostragens para as análises da densidade básica, poder calorífico superior, teor de cinzas da madeira e foi estimado o índice de valor combustível. Considerando os valores das análises realizadas e do volume em madeira, foram determinadas as quantidades de energia estocada por espécie e por área. O experimento foi avaliado segundo o delineamento inteiramente casualizado, com 5 tratamentos (espécies) na área I, 6 na área II e 12 na área III, com três repetições em cada área. A madeira da espécie Croton sonderianus apresentou para as três áreas maior valor de poder calorífico e a Mimosa tenuiflora maior valor de densidade básica e poder calorifico nas áreas I e II. O mesmo ocorreu para a Caesalpinia leiostachya, Caesalpinia pyramidalis, Piptadenia moniliformis, Pilosocereus pachycladus e a não identificada na área III, o que resultou na maior densidade energética nesta área quando comparadas às demais. Dessa maneira, a comercialização da madeira para fins energéticos deve ser com base na densidade energética que determinada área sob manejo florestal possui e não somente no volume de madeira.

Palavras-chave: Biomassa; Caatinga; Lenha; Qualidade da madeira.

\section{Abstract}

The objective of this work was to determine the energy density in three different areas under sustainable forest management in Rio Grande do Norte (RN). From the access to the forest inventory of each area, the selection of the species with $70 \%$ of representativity based on the importance value index (IVI) was carried out, as well as the knowledge about the real volume of wood. From each species,

Fonte de financiamento: Coordenação de Aperfeiçoamento de Pessoal de Nível Superior - Brasil (CAPES) - Código Financeiro 001.

Conflito de interesse: Nada a declarar.

Autor correspondente: cynthiapss@live.com

Recebido: 18 setembro 2018.

Aceito: 4 julho 2019.

Editor: Francides Gomes Silva Júnior

Este é um artigo publicado em acesso aberto (Open Access) sob a licença Creative Commons Attribution, que permite uso, distribuição e reprodução em qualquer meio, sem restrições desde que o trabalho original seja corretamente citado. 
three trees with characteristics that represented each stand were harvested, and samples were taken for the analyzes of basic density, upper calorific value, ash content of the wood and the value of fuel value was estimated. Considering the values of the analyzes performed and the volume of wood, the quantity of energy stored by species and area was determined. The experiment was evaluated according to a completely randomized design, with 5 treatments (species) in area I, 6 in area II and 12 in area III, with three replicates in each area. The wood of the species Croton sonderianus presented a higher value of calorific value for the three areas and the Mimosa tenuiflora had a higher value of basic density and heat power in areas I and II. The same occurred for Caesalpinia leiostachya, Caesalpinia pyramidalis, Piptadenia moniliformis, Pilosocereus pachycladus and unidentified in area III, which resulted in a higher energy density in this area when compared to the others. In this way, the commercialization of the wood for energy purposes must be based on the energy density that a certain area under forest management has and not only on the volume of wood.

Keywords: Biomass; Caatinga; Firewood; Wood quality.

\section{INTRODUÇÃO}

A demanda por energéticos florestais (lenha e carvão) na região Nordeste do Brasil é representada pelo consumo industrial e comercial estimado entre 25 e 30 milhões de estéreos de madeira por ano, e em 2017 a lenha foi responsável por 7,8\% da produção de energia primária (Riegelhaupt \& Pareyn, 2010; Empresa de Pesquisa Energética, 2018). Nessa região, a principal oferta de madeira é obtida de espécies florestais nativas do bioma Caatinga, que fornece combustível para fornos de indústrias e comércios (Lima Júnior et al., 2014).

Há preocupação com a extração da madeira da Caatinga, pois se acredita que $50 \%$ da cobertura florestal original foi reduzida (Almeida et al., 2015). As demandas por madeira de espécies nativas podem ser supridas de forma sustentável pela adoção de práticas como a exploração florestal sob o manejo sustentável.

Dessa forma, tal prática é idealizada por conjunto de técnicas que visam rotacionar a exploração em determinada área, a considerar a particularidade das características de cada floresta (Andrade et al., 2019). No Rio Grande do Norte, a Lei Florestal 6.679/1995 estabelece que qualquer exploração dos recursos da floresta nativa só pode ser realizada mediante o Plano de Manejo Florestal de Rendimento Sustentável (Rio Grande do Norte, 1995). Segundo Carvalho (2018), até o início do ano de 2018, o Rio Grande do Norte possuía 21 planos de manejo com licença vigente.

Embora o inventário, que é parte importante na instalação de áreas sob manejo, contemple informações sobre o rendimento volumétrico de biomassa florestal, a densidade energética não é estimada e nem considerada para determinar seu potencial para exploração.

Contudo, é importante considerar a associação da análise do rendimento em madeira à avaliação da qualidade desta para energia na caracterização de espécies florestais, seja para queima direta (combustão) ou para a produção de carvão vegetal, de modo que ocorra o planejamento correto da exploração desse recurso (Santos et al., 2013; Lima Júnior et al., 2015). Também não há considerações importantes como a estimativa da produção de energia em diferentes áreas sob manejo, em razão da diversidade de espécies presentes em um plano que, de acordo com Pareyn et al. (2015), esperam-se diferentes respostas da vegetação manejada em áreas distintas.

Dessa forma, para atender a demanda de madeira como fonte energética é importante conhecer a densidade energética, que pode ser fornecida por espécies presentes em área sob manejo florestal. Dentro deste contexto, o objetivo deste trabalho foi determinar o índice de valor combustível e a densidade energética de espécies que ocorrem em três diferentes áreas sob manejo, localizadas no Estado do Rio Grande do Norte (RN). 


\section{MATERIAL E MÉTODOS}

\section{Localizações e descrições das áreas de estudo}

Para a realização do presente estudo foram utilizadas madeiras provenientes de três áreas sob regime de manejo florestal localizados no interior do estado do Rio Grande do Norte. O plano de manejo da Fazenda Belo Monte (área I) possui sete anos de exploração, com ciclo de corte de 16 anos. Localiza-se no município de Campo Grande/RN, sob coordenadas $05^{\circ} 52^{\prime} 10,1^{\prime \prime} \mathrm{S}$ e $37^{\circ} 18^{\prime} 44,4^{\prime \prime} \mathrm{W}$, dispondo de uma área sob manejo de 901.16 hectares com rendimento médio de 169.89 st/ha. Segundo a classificação de Köppen, o clima é do tipo BSw'h', a tipologia de solo predominante é a Bruno Não Cálcico e a precipitação média anual é de 400 a $750 \mathrm{~mm}$, com período chuvoso entre os meses de fevereiro a maio (Carvalho, 2010).

A área II refere-se ao plano de manejo da Fazenda Estrela do Norte que se situa no município de Cruzeta/RN, sob coordenadas 6¹6'34.88" S e 3648'43.65"W. Apresenta área manejada de 777,42 ha e rendimento médio de 137 st/ha. Está em seu oitavo ano de exploração e possui ciclo de corte com planejamento para 15 anos. O clima é classificado como muito seco e semi-árido, a pluviosidade média anual é cerca de 588,8 mm, com período chuvoso de fevereiro a abril e o solo é do tipo latossolo vermelho-amarelo (Lopes, 2008).

A Fazenda São Pedro (área III), localiza-se no município de Touros/RN, sob coordenadas $5^{\circ} 25^{\prime} 19,64^{\prime \prime} \mathrm{S}$ e $35^{\circ} 45^{\prime} 30,32^{\prime \prime}$ W. Segundo Carvalho (2009), o plano de manejo possui oito anos de exploração, com ciclo de corte de 15 anos em 600 ha de caatinga arbustiva arbórea manejada e rendimento médio de 207.16 st/ha. O clima segundo a classificação de Köppen é do tipo As', a precipitação pluviométrica anual média é de $648,6 \mathrm{~mm}$ e ocorrem dois tipos de solos: areias quartzosas distróficas e vertissolos. Na Figura 1 estão indicadas, em destaque, as localizações das três áreas de estudo.

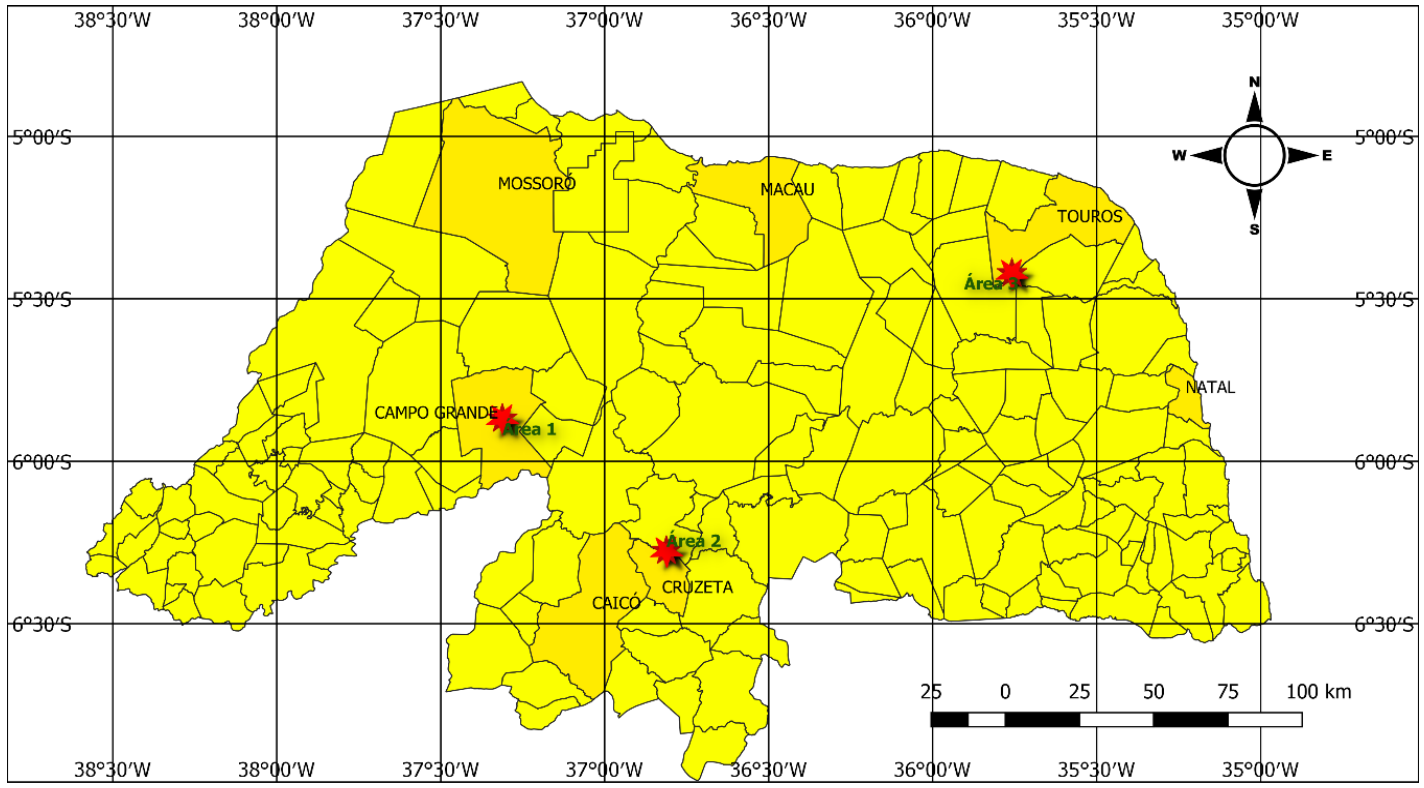

Figura 1. Localização das áreas de estudo, dentro do estado do Rio Grande do Norte.

\section{Seleção das espécies}

As espécies foram selecionadas em função do levantamento fitossociológico, conforme informações acessadas no inventário florestal, a partir do índice de valor de importância (IVI). O cálculo do IVI foi realizado a partir da consideração dos dados de densidade, dominância e frequência relativa destas espécies, conforme Souza \& Soares (2013). 


\section{Amostragem}

De cada espécie selecionada foram abatidas três árvores com boas condições fitossanitárias, livre do efeito bordadura e representativas quanto ao diâmetro médio do povoamento. De cada árvore retirou-se cinco discos de aproximadamente $10 \mathrm{~cm}$ de espessura, nas seguintes posições: o primeiro na base (a $30 \mathrm{~cm}$ do solo) e os demais a $25 \%$ de distância entre eles, até atingir três centímetros de diâmetro do fuste principal. Para as árvores bifurcadas abaixo da altura do peito (1.30m de altura do solo) e com mais de um fuste, foi selecionado o fuste central para a retirada dos discos (Figura 2).

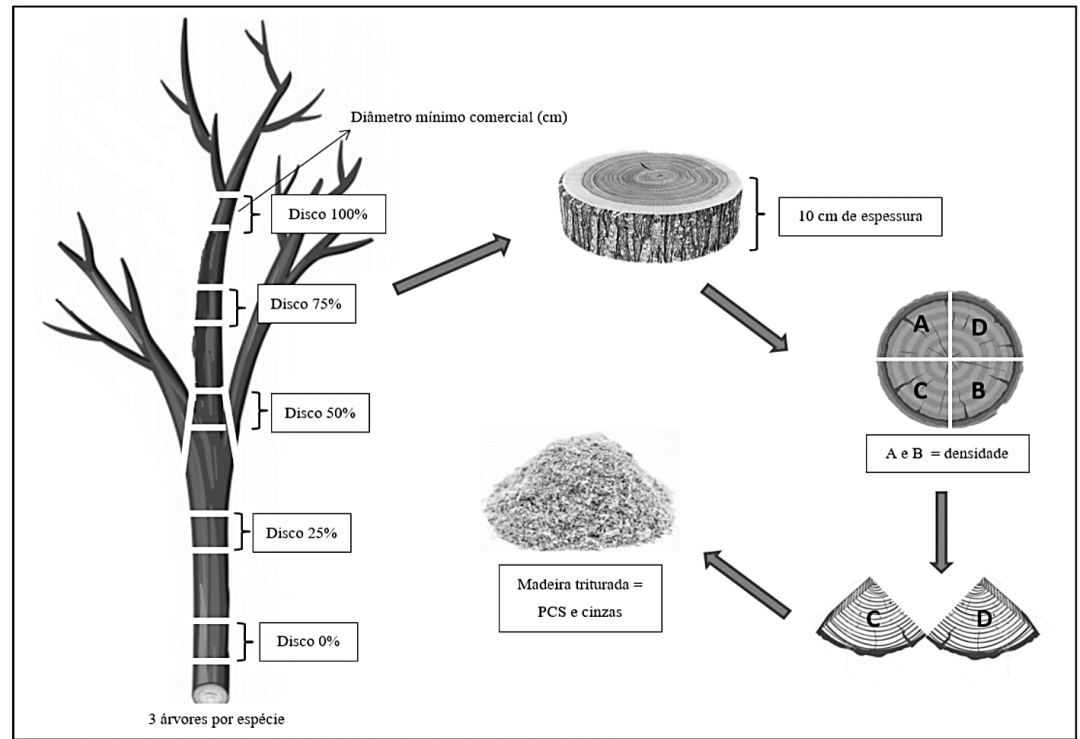

Figura 2. Método de coleta das amostras para a realização das análises. Fonte: o autor.

\section{Preparo das amostras}

Inicialmente foram retiradas as cascas dos discos, e em seguida esses foram subdivididos em cunhas opostas passando pela medula. Duas dessas foram destinadas à determinação da densidade básica da madeira e as outras duas, encaminhadas para trituração e adequação à granulometria referida nas respectivas normas técnicas, e posteriormente, foi realizada uma amostragem composta do material por tratamento, para determinação do poder calorífico superior e teor de cinzas da madeira (Figura 2).

\section{Parâmetros avaliados}

A análise da densidade básica da madeira foi realizada conforme o método de imersão em água, descrito na norma NBR 11941 da Associação Brasileira de Normas Técnicas (2003) e, a partir de uma bomba calorimétrica adiabática determinou-se o poder calorífico superior conforme as recomendações da norma ASTM D2015-1996 (American Society for Testing and Materials, 2000). Para estimar o teor de cinzas de cada espécie selecionada, utilizou-se uma mufla, em que a análise foi realizada conforme a metodologia descrita na norma ASTM E1755-2001 (American Society for Testing and Materials, 2007). Após a determinação dos fatores que interferem na qualidade da madeira para a geração de energia, foi realizado o cálculo do índice de valor combustível (IVC) adaptado ao proposto por Purohit \& Nautiyal (1987), de acordo com a Equação 1.

$$
I V C=\frac{P C S^{*} D B}{T C}
$$

em que, PCS é o Poder calorífico superior (KJ/g); DB, a Densidade básica da madeira $\left(\mathrm{g} / \mathrm{cm}^{3}\right)$ e TC, o Teor de cinzas (\%).

Para os cálculos da produção de energia, as informações sobre o volume real em lenho por espécie em cada área, foram obtidas a partir do inventário florestal. A partir da 
multiplicação entre a densidade básica $\left(\mathrm{kg} \cdot \mathrm{m}^{-3}\right)$ e o poder calorífico superior $\left(\mathrm{kcal}^{\mathrm{k}} \mathrm{kg}^{-1}\right)$ foi determinada a produção de energia em $\mathrm{kcal} / \mathrm{m}^{3}$. A fim de obter o resultado em $\mathrm{kcal} / \mathrm{ha}$ multiplicou-se a energia produzida por metro cúbico de madeira $\left(\mathrm{kcal} / \mathrm{m}^{3}\right)$ pela produtividade média de cada espécie $\left(\mathrm{m}^{3} \cdot \mathrm{ha}^{-1}\right)$, de acordo com a metodologia utilizada por Santos et al. (2013).

\section{Análises estatísticas}

O experimento foi avaliado segundo o delineamento inteiramente casualizado, sendo 5 tratamentos (espécies selecionadas com base no IVI) para área I, 6 tratamentos (espécies selecionadas com base no IVI) para área II e 12 tratamentos (espécies selecionadas com base no IVI) para área III, com três repetições (árvores-amostra) por área. Os dados foram submetidos aos testes de Lilliefors para testar a normalidade e Cochran para testar a homogeneidade das variâncias. Em seguida, procedeu-se à análise de variância pelo teste $F$, sendo as médias comparadas pelo Skott-Knott. Considerou-se o nível de significância de 5\%. As análises estatísticas foram realizadas com o auxílio do software R Core Team (2018).

\section{RESULTADOS E DISCUSSÃO}

Conforme o índice de valor de importância das espécies, foram identificadas cinco espécies florestais na área I, seis espécies florestais na área II e doze espécies florestais na área III, que representaram pelo menos $70 \%$ do IVI de todo povoamento florestal dos planos de manejo florestal sustentáveis estudados (Tabela 1).

Tabela 1. Espécies selecionadas para amostragem seguidas de seus valores de índice de valor de importância, percentual representativo no povoamento florestal por área e volume do lenho produzido em $\mathrm{m}^{3} \cdot \mathrm{ha}^{-1}$.

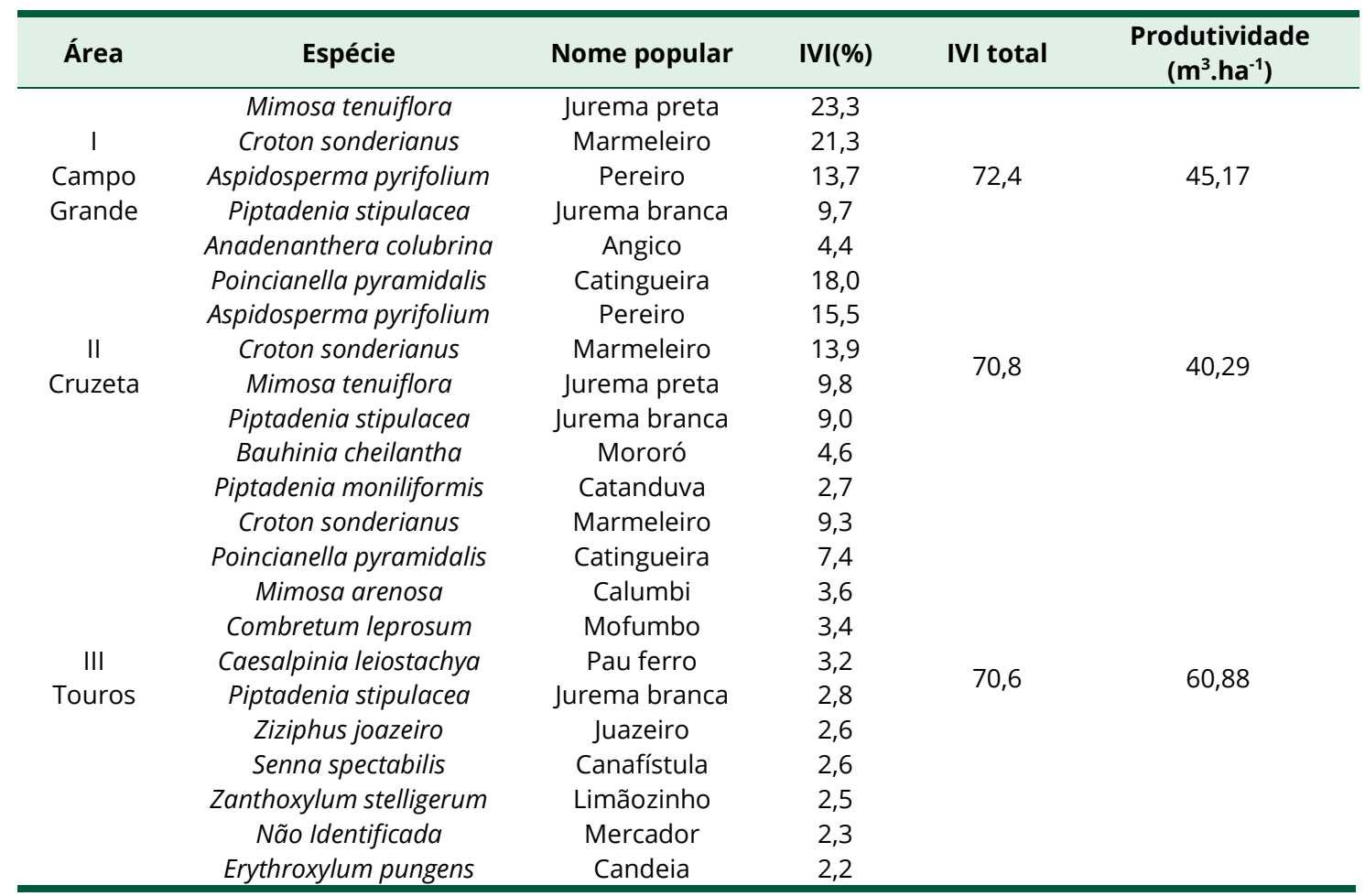

*IVI: índice de valor de importância. Fonte: PMFS Fazenda Belo Monte 2010; PMFS Fazenda Estrela do Norte 2008; PMFS fazenda São Pedro 2009.

Embora a fazenda situada na região de Campo Grande possua maior extensão de área sob manejo $(901,16$ ha), observa-se que a diversidade de espécies representativas é menor quando comparada ao plano de manejo de Touros que possui menor extensão (600 ha). 
Segundo Brand (2017) quanto maior a diversidade de espécies florestais presentes na área, maior será a variação dos parâmetros da qualidade da madeira com finalidade para uso energético, como a densidade básica e o poder calorífico superior.

A madeira destinada à geração de energia é comercializada com base no volume lenhoso, o que não considera a quantidade de massa existente em certo volume de madeira. Dessa maneira, nota-se que a área III possui a maior produtividade em volume. No entanto, como a madeira é utilizada como combustível, as considerações da quantidade de energia potencial para oferta devem ser estimadas, pois as espécies com menos volume na área podem não contribuir significativamente com a produtividade de energia por ha. Para isso, deve-se conhecer as características da madeira que determinam a densidade energética.

$\mathrm{Na}$ Tabela 2 estão apresentados os valores médios de densidade básica $\left(\mathrm{kg} \cdot \mathrm{m}^{-3}\right)$, poder calorífico superior $\left(\mathrm{kcal}_{\mathrm{kg}}{ }^{-1}\right)$, teor de cinzas e índice de valor combustível das madeiras das espécies selecionadas para o estudo, por área.

Tabela 2. Densidade básica, poder calorífico superior, teor de cinzas e índice de valor combustível das madeiras de espécies nativas amostradas em cada área sob manejo florestal sustentável estudada.

\begin{tabular}{|c|c|c|c|c|c|}
\hline Área & Espécie & $\begin{array}{c}\text { DB } \\
\left(\mathrm{kg} \cdot \mathrm{m}^{-3}\right)\end{array}$ & $\begin{array}{c}\text { PCS } \\
\left(\mathrm{kcal} . k g^{-1}\right)\end{array}$ & TC (\%) & IVC \\
\hline & Mimosa tenuiflora & $799 a$ & $4830 a$ & $0,8 \mathrm{c}$ & $22,43 a$ \\
\hline I & Croton sonderianus & $630 \mathrm{~b}$ & $4800 \mathrm{a}$ & $0,5 \mathrm{c}$ & $30,74 a$ \\
\hline Campo & Aspidosperma pyrifolium & $634 b$ & $4823 \mathrm{a}$ & $1,4 \mathrm{~b}$ & $9,85 \mathrm{~b}$ \\
\hline \multirow[t]{4}{*}{ Grande } & Piptadenia stipulacea & $716 a$ & $4748 \mathrm{~b}$ & $1,0 \mathrm{c}$ & $13,92 b$ \\
\hline & Anadenanthera colubrina & $710 a$ & $4653 \mathrm{c}$ & $2,8 \mathrm{a}$ & $4,98 \mathrm{~b}$ \\
\hline & Poincianella pyramidalis & $718 c$ & $4504 \mathrm{c}$ & $4,6 \mathrm{a}$ & $3,09 \mathrm{~b}$ \\
\hline & Aspidosperma pyrifolium & $678 d$ & $4873 \mathrm{a}$ & $1,0 \mathrm{~b}$ & $15,01 \mathrm{a}$ \\
\hline ॥ & Croton sonderianus & $654 d$ & $4786 a$ & $0,6 \mathrm{~b}$ & $21,74 a$ \\
\hline \multirow[t]{7}{*}{ Cruzeta } & Mimosa tenuiflora & $839 a$ & $4832 \mathrm{a}$ & $1,0 \mathrm{~b}$ & $17,85 \mathrm{a}$ \\
\hline & Piptadenia stipulacea & $701 \mathrm{c}$ & $4676 \mathrm{~b}$ & $1,4 \mathrm{~b}$ & $12,13 \mathrm{a}$ \\
\hline & Bauhinia cheilantha & 798 b & 4793 a & $2,0 \mathrm{~b}$ & $7,99 \mathrm{~b}$ \\
\hline & Piptadenia moniliformis & $750 a$ & $4726 a$ & $1,2 \mathrm{c}$ & $13,15 b$ \\
\hline & Croton sonderianus & $638 \mathrm{~b}$ & $4813 a$ & $1,3 \mathrm{c}$ & $10,40 \mathrm{~b}$ \\
\hline & Poincianella pyramidalis & $791 \mathrm{a}$ & 4559 c & $2,3 b$ & $6,72 b$ \\
\hline & Mimosa arenosa & $830 a$ & $4700 \mathrm{~b}$ & $1,6 \mathrm{c}$ & $10,35 \mathrm{~b}$ \\
\hline III & Combretum leprosum & $699 \mathrm{~b}$ & $4760 \mathrm{a}$ & $2,1 \mathrm{~b}$ & $6,94 \mathrm{~b}$ \\
\hline \multirow[t]{7}{*}{ Touros } & Caesalpinia leiostachya & $771 \mathrm{a}$ & $4809 a$ & $0,9 \mathrm{c}$ & $23,06 a$ \\
\hline & Piptadenia stipulacea & $647 b$ & $4667 \mathrm{~b}$ & $2,1 \mathrm{~b}$ & $6,96 \mathrm{~b}$ \\
\hline & Ziziphus joazeiro & $659 \mathrm{~b}$ & 4562 c & $2,7 \mathrm{a}$ & $4,70 \mathrm{~b}$ \\
\hline & Senna spectabilis & $496 \mathrm{c}$ & $4761 \mathrm{a}$ & $0,8 \mathrm{c}$ & $12,46 \mathrm{~b}$ \\
\hline & Zanthoxylum stelligerum & $722 \mathrm{a}$ & $4671 \mathrm{~b}$ & $1,6 \mathrm{c}$ & $9,02 \mathrm{~b}$ \\
\hline & Não Identificada & $793 a$ & $4631 \mathrm{~b}$ & $1,4 \mathrm{c}$ & $11,64 b$ \\
\hline & Erythroxylum pungens & $674 \mathrm{~b}$ & 4832 a & $1,9 \mathrm{~b}$ & $7,47 \mathrm{~b}$ \\
\hline
\end{tabular}

DB = densidade básica, PCS = poder calorífico superior, TC = teor de cinzas, IVC = índice de valor combustível. Médias seguidas da mesma letra entre as espécies, para uma mesma área e variável, não diferem entre si, a 5\% de significância, pelo teste Skott-Knott.

Em estudo desenvolvido com sete espécies florestais da Caatinga de extrato arbóreo do Rio Grande do Norte, realizado por Santos et al. (2016), identificou resultados superiores da densidade básica da madeira Bauhinia cheilantha $\left(970 \mathrm{~kg} \cdot \mathrm{m}^{-3}\right)$ e Mimosa tenuiflora $\left(900 \mathrm{~kg} \cdot \mathrm{m}^{-3}\right)$ as quais os autores classificaram como as mais densas.

As madeiras das espécies Mimosa tenuiflora (jurema preta), Piptadenia stipulacea (jurema branca), Anadenanthera colubrina (angico), Piptadenia moniliformis (catanduva), Poincianella pyramidalis (catingueira), Mimosa arenosa (calumbi), Caesalpinia leiostachya (pau ferro), Zanthoxylum stelligerum (limãozinho) e a não identificada (mercador) apresentaram, os maiores valores médios de densidade básica, de acordo com a área que estão presentes. Em cada plano de manejo, o conhecimento da qualidade da madeira e das espécies potenciais possibilita a comercialização desse combustível conforme a sua potencialidade, não se restringindo apenas à venda segundo o volume de madeira produzido. Santos et al. (2013), afirmam que a densidade básica, entre outros parâmetros, são considerações imprescindíveis para o uso e indicação deste recurso como fonte de energia. 
Apesar da comparação dos resultados serem entre as espécies de uma mesma área, é possível evidenciar que a variação de sítio como as condições de clima, precipitação e solo, além da idade do povoamento, podem influenciar diretamente nas características da madeira, a exemplo da densidade básica (Morais et al., 2017). Isso pode ser observado para a densidade básica da madeira de Mimosa tenuiflora que possui $799 \mathrm{~kg} \cdot \mathrm{m}^{-3}$ na área I e de $839 \mathrm{~kg} \cdot \mathrm{m}^{-3}$ na área II, a Piptadenia stipulacea que apresenta $701 \mathrm{~kg} \cdot \mathrm{m}^{-3}$ na área II e $647 \mathrm{~kg} \cdot \mathrm{m}^{-3}$ na área III.

Para determinar um combustível ideal é imprescindível a consideração das variáveis da madeira que influenciam no processo de queima. A identificação do valor combustível é mensurada pelo índice de valor combustível que considera a densidade básica e o poder calorífico da madeira como características positivas e o teor de cinzas como característica negativa.

Observa-se que o índice valor combustível varia para uma mesma espécie, como por exemplo, o Croton sonderianus, que apresentou IVC cerca de três vezes maior na área I em relação ao observado na área III (Tabela 2). Este mesmo fato foi observado com a Piptadenia stipulacea que na área I apresentou IVC duas vezes maior que na área III.

O índice de valor combustível é parâmetro fundamental para indicar o uso energético da madeira de uma espécie, pois pondera as características positivas e negativas desse combustível e, dessa forma, os valores comparativos intermediários e maiores são desejáveis para esse parâmetro (Baqir et al., 2017). Assim, o teor de cinzas afetou significativamente os resultados apresentados e, algumas espécies que obtiveram altos valores de PCS e densidade básica, divergiram estatisticamente do melhor resultado obtido para o IVC, como a Piptadenia stipulacea e Aspidosperma pyrifolium na área I, Bauhinia cheilantha na área II e todas as espécies com exceção da Caesalpinia leiostachya na área III.

Ramos et al. (2008), avaliando o potencial do uso combustível de madeiras da Caatinga, observou valores do índice de valor combustível das espécies semelhantes ao aqui estudado como o Croton sonderianus (30.9), Poincianella pyramidalis $(26,6)$, Anadenanthera colubrina $(26,0)$, Bauhinia cheilantha (22.8), Piptadenia stipulacea $(22,4)$ e Mimosa tenuiflora $(22,6)$.

Embora o IVC identifique as espécies com melhores características energéticas, o estoque de energia em uma área sob manejo é determinado de acordo com a qualidade da madeira de todas as espécies nela inseridas. A Figura 3 mostra os valores estimados da quantidade de energia em $\mathrm{Mkcal} / \mathrm{m}^{3}$ estocada na madeira de cada espécie por área.

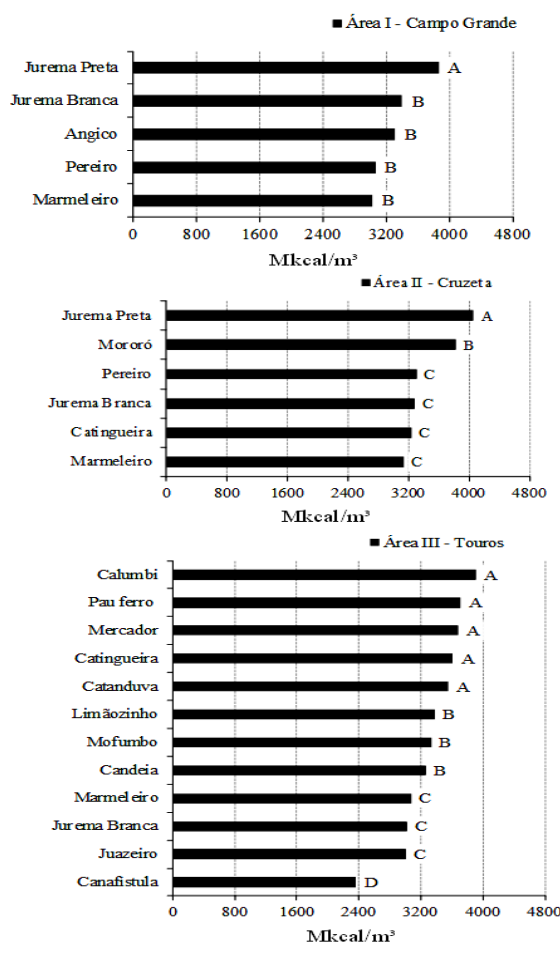

Figura 3. Densidade energética em $\mathrm{Mkcal} / \mathrm{m}^{3}$ nas madeiras das espécies estudadas, por área 
Verificou-se que a maior densidade energética foi observada na madeira da espécie Mimosa tenuiflora (jurema preta) nas áreas I e II. Vale ressaltar que a representatividade (IVI) dessa espécie foi maior na área I (23,3\%). No entanto, não se observa o mesmo em relação ao IVI na área II $(9,8)$.

Torna-se importante considerar os resultados observados uma vez que se entende que a produção em $\mathrm{m}^{3}$ (volume) deve estar associada à qualidade da madeira, de modo que se estime a produção de energia com base na identificação das espécies potenciais para tal fim (Montoya, 2017). Dessa maneira, a comercialização da madeira proveniente do manejo florestal deveria ser selecionada com base na maior produção de biomassa pelas espécies potencialmente energéticas e a conservação ou utilização para outras finalidades das menos potenciais (Montoya, 2017). Os valores da produtividade de energia da madeira, em Mkcal/ha, de cada área avaliada, estão apresentados na Figura 4.

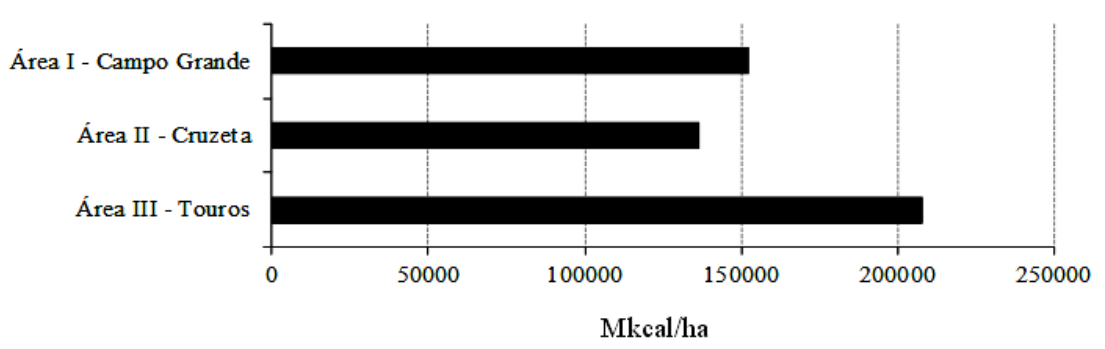

Figura 4. Produtividade de energia em Mkcal/ha de cada área sob manejo estudada.

Observou-se que a área III além de apresentar maior rendimento volumétrico em lenho (207 st/ha) quando comparada às áreas I (169,89 st/ha) e II (137 st/ha) obteve maior riqueza na ocorrência de espécies com grande densidade energética na madeira, fator esse que responde a alta produtividade em $\mathrm{kcal} / \mathrm{ha}$ frente as demais áreas estudadas. A representação da madeira da Piptadenia moniliformis (catanduva) se destaca por possuir maiores valores de densidade básica e poder calorífico superior e apresentar nesta área uma representatividade de $28.7 \%$, superior às demais porcentagens de IVI das outras espécies.

\section{CONCLUSÕES}

Concluiu-se que para as áreas I e II as madeiras das espécies Mimosa tenuiflora e Croton sonderianus apresentaram os maiores IVC. As madeiras de Aspidosperma pyrifolium e Piptadenia stipulacea apresentaram valores do teor cinzas maiores, em comparação as demais espécies, o que inibiu um desempenho satisfatório para o IVC. Em relação à área III a madeira da Caesalpinia leiostachya destacou-se com o maior IVC. Os maiores valores para DB e PCS encontrados para a Mimosa tenuiflora nas áreas I e II, colaboraram com a maior densidade energética de madeira por espécie. Já para a área III esses resultados foram observados para as espécies Mimosa arenosa, Caesalpinia leiostachya, a não identificada (mercador), Poincianella pyramidalis e Piptadenia moniliformis. Ao se considerar a produtividade energética por área, o destaque foi para a área III, por esta apresentar maior número de espécies representativas quanto a densidade energética da madeira, seguida da área I e II.

A comercialização da madeira para fins energéticos deve ser com base na densidade energética (estoque de energia) presente na área sob manejo e não somente com base na produção em volume de lenho, pois além do volume as características da madeira para tal fim influenciarão diretamente no estoque de energia presente na área. 


\section{REFERÊNCIAS}

Almeida, A. M. C., Oliveira, E., Calegari, L., Medeiros Neto, P. N., \& Pimenta, A. S. (2015). Avaliação físicoquímica e energética da madeira das espécies Piptadenia stipulacea (Benth.) Ducke e Amburana cearensis (Allemao) A. C. Smith de ocorrência no semiárido nordestino brasileiro. Ciência Florestal, 25(1), 165-173. http://dx.doi.org/10.5902/1980509817474.

American Society for Testing and Materials - ASTM. (2000). Standard D2015-1996: standard test method for gross calorific value of coal and coke by the adiabatic bomb calorimeter. West Conshohocken: ASTM International.

American Society for Testing and Materials - ASTM. (2007). Standard E1755-2001: standard test method for ash in biomass. West Conshohocken: ASTM International.

Andrade, F. N., Lopes, J. B., Barros, R. F. M., \& Lopes, C. G. R. (2019). Caracterização dos planos de manejo florestal no estado do Piauí. Ciência Florestal, 29(1), 243-254. http://dx.doi.org/10.5902/1980509827998.

Associação Brasileira de Normas Técnicas - ABNT. (2003). ABNT NBR 11491: madeira: determinação da densidade básica. Rio de Janeiro: ABNT. 6 p.

Baqir, M., Kothari, R., \& Singh, R. P. (2017). Characterization and ranking of subtropical trees in a rural plantation forest of Uttar Pradesh, India, as fuel wood using fuel wood value index (FVI). Environment, Development and Sustainability, 21(2), 763-776. http://dx.doi.org/10.1007/s10668-0170057-z.

Brand, M. A. (2017). Potencial de uso da biomassa florestal da Caatinga, sob manejo sustentável, para geração de energia. Ciência Florestal, 27(1), 117-127. http://dx.doi.org/10.5902/1980509826452.

Carvalho, A. C. (2018). Potencial energético da madeira de espécies florestais em área sob manejo sustentável, após corte raso, no Rio Grande do Norte (Dissertação de mestrado). Programa de Pósgaduação em Ciências Florestais, Universidade Federal do Rio Grande do Norte, Macaíba.

Carvalho, A. J. E. (2009). Plano de manejo florestal fazenda São Pedro. Natal: Florescer Projetos e Consultoria Ambiental Ltda.

Carvalho, A. J. E. (2010). Plano de manejo florestal fazenda Belo Monte. Natal: Florescer Projetos e Consultoria Ambiental Ltda.

Empresa de Pesquisa Energética - EPE. (2018). Balanço Energético Nacional 2018: ano base 2017. Rio de Janeiro: EPE. Recuperado em 12 de junho de 2018, de http://epe.gov.br/pt/publicacoes-dadosabertos/publicacoes/balanco-energetico-nacional-2018

Lima Júnior, C., Lima, R. L. F., Liberal, B. G., Guerrero, J. R. H., Sampaio, E. V. S. B., \& Menezes, R. S. C. (2015). Viabilidade econômica do uso energético de lenha da caatinga sob manejo sustentável. Revista Brasileira de Geografia Física, 8(1), 156-166. http://dx.doi.org/10.26848/rbgf.v8i1.233582.

Lima Júnior, C., Sampaio, E. V. S. B., Lima, R. F. A., \& Menezes, R. S. C. (2014). Potencial de aproveitamento energético de fontes de biomassa no nordeste do Brasil. Revista Brasileira de Geografia Física, 7(2), 207-221. Recuperado em 6 de junho de 2018, de https://periodicos.ufpe.br/revistas/rbgfe/article/view/233046/26969

Lopes, E. W. F. (2008). Plano de manejo florestal sustentável: fazenda Estrela do Norte. Cruzeta: Agropecuária Estrela do Norte Ltda.

Montoya, J. D. M. (2017). Potencial energético de espécies lenhosas da caatinga para uso de biomassa em plano de manejo florestal (Dissertação de mestrado). Programa de Pós-graduação em Ciências Florestais, Universidade Federal Rural de Pernambuco, Recife.

Morais, P. H. D., Longue Júnior, D., Colodette, J. L., Morais, E. H. C., \& Jardim, C. M. (2017). Influence of clone harvesting age of Eucalyptus grandis and hybrids of Eucalyptus grandis x Eucalyptus urophylla in the wood chemical composition and in kraft pulpability. Ciência Florestal, 27(1), 237-248. http://dx.doi.org/10.5902/1980509826462.

Pareyn, F. G. C., Pereira, W. E., Salcedo, I. H., Riegelhaupt, E., Gomes, E. C., \& Cruz Filho, J. L. V. (2015). A influência da precipitação sobre o crescimento e os ciclos de corte da caatinga manejada: uma primeira aproximação. In F. Pareyn, J. L. Vieira \& A. Gariglio (Eds.), A influência da precipitação sobre o crescimento e os ciclos de corte da caatinga manejada: uma primeira aproximação (1. ed., Vol. 2, pp. 30-39). Recife: Associação Plantas do Nordeste.

Purohit, N., \& Nautiyal, A. R. (1987). Fuelwood value index of indian mountain tree species. International Tree Crops Journal, 4(2-3), 177-182. http://dx.doi.org/10.1080/01435698.1987.9752821.

R Core Team. (2018). $R$ : a language and environment for statistical computing. Vienna: R Foundation for Statistical Computing. Recuperado em 12 de maio de 2018, de https://www.R-project.org/ 
Ramos, M. A., Medeiros, P. M., Almeida, A. L. S., Feliciano, A. L. P., \& Albuquerque, U. P. (2008). Can wood quality justify local preferences for firewood in an area of caatinga (dryland) vegetation? Biomass and Bioenergy, 32(6), 503-509. http://dx.doi.org/10.1016/j.biombioe.2007.11.010.

Riegelhaupt, E. M. E., \& Pareyn, F. G. C. (2010). A questão energética. In M. A. Gariglio, E. V. S. B. Sampaio, L. A. Cestaro \& P. Y. Kageyama (Eds.), Uso sustentável e conservação dos recursos florestais da Caatinga (pp. 65-77). Brasília: Serviço Florestal Brasileiro.

Rio Grande do Norte. (1995). Lei n 6.679, de 1995. Dispõe sobre a Política Florestal do Estado do Rio Grande do Norte e dá outras providências. Diário Oficial do Estado do Rio Grande do Norte, Natal.

Santos, R. C., Carneiro, A. C. O., Pimenta, A. S., Castro, R. V. O., Marinho, I. V., Trugilho, P. F., Alves, I. C. N., \& Castro, A. F. N. M. (2013). Potencial energético da madeira de espécies oriundas de plano de manejo florestal no Estado do Rio Grande do Norte. Ciência Florestal, 23(2), 491-502. http://dx.doi.org/10.5902/198050989293.

Santos, R. C., Castro, R. V. O., Carneiro, A. D. C. O., Castro, A. F. N. M., Pimenta, A. S., Pinto, E. M., \& Marinho, I. V. (2016). Estoques de volume, biomassa e carbono na madeira de espécies da Caatinga em Caicó, RN. Pesquisa Florestal Brasileira, 36(85), 1-7, 31. http://dx.doi.org/10.4336/2016.pfb.36.85.772.

Souza, A. L., \& Soares, C. P. B. (2013). Florestas nativas: estrutura, dinâmica e manejo. Viçosa: Editora UFV.

Contribuição dos autores: CPSS: conceituação, curadoria de dados, análise formal, obtenção de financiamento, investigação, metodologia, administração do projeto, recursos, software, supervisão, validação, visualização, escrita - primeira redação, escrita - revisão e edição; RCS: obtenção de financiamento, investigação, administração do projeto, recursos, supervisão, validação, escrita - revisão e edição; AJEC: conceituação, curadoria de dados, metodologia, recursos; RVOC: curadoria de dados, análise forma, software, supervisão, validação, visualização, escrita - revisão e edição; SELC: conceituação, metodologia, visualização, escrita - primeira redação, escrita - revisão e edição; LIL: análise formal, investigação, metodologia, recursos, escrita - primeira redação; FGCP: conceituação, investigação, metodologia, recursos, supervisão, validação, visualização; AFDJ: curadoria de dados, análise formal, metodologia, software, supervisão, validação, visualização, escrita - revisão e edição; PFT: conceituação, análise formal, supervisão, validação, visualização, escrita - revisão e edição; NFOC: conceituação, metodologia, escrita - primeira redação; MAM: curadoria de dados, análise formal, software. 